\title{
Reverse epidemiology of elevated blood pressure among chronic hemodialysis black patients with stroke: a historical cohort study
}

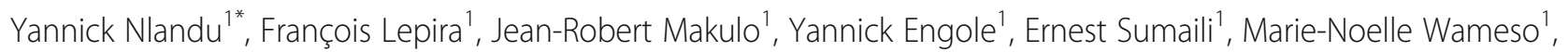
Vieux Mokoli ${ }^{1}$, Jeannine Luse ${ }^{2}$, Augustin Longo ${ }^{1}$, Chantal Zinga ${ }^{1}$, Pierre Akilimali ${ }^{3}$, Aliocha Nkodila', Mélanie Bavassa ${ }^{4}$, François Kajingulu ${ }^{1}$, Justine Bukabau ${ }^{1}$ and Nazaire Nseka ${ }^{1}$

\begin{abstract}
Background: Stroke is the third leading cause of cardiovascular mortality in dialysis patients. The objective of this study was to assess the extent of stroke in chronic hemodialysis patients.

Methods: Historical cohort of patients enrolled in two hemodialysis (HD) centers from January 1, 2010 to December 31, 2011, including 191 patients (mean age 52 years, 68\% men). Incidence curves and survival time analysis between the first day of HD and the end of the study were described by the Kaplan-Meier method. Independent stroke predictors were identified by multiple logistic regression analysis. $P<0.05$ defined the level of statistical significance.
\end{abstract}

Results: 12 incident stroke were recorded during the study period, with 1622.1 person-months (PM), a stroke incidence rate of 7.4 cases per 1000 PM $(95 \% \mathrm{Cl}=7.35-7.44)$ at the point date. The incidence of stroke at 6 months, 12 months and 24 months was 9.8\%, 11.9\% and 13\%, respectively. Only the absence of arterial hypertension $(\mathrm{RR}=5.7,95 \% \mathrm{Cl}: 1.52-21.42)$ emerged as an independent determinant of stroke.

Conclusion: The high incidence of stroke in Kinshasa HD centers is partially explained by reverse epidemiology. Efforts must be made to understand this phenomenon in order to reduce its impact.

Keywords: Stroke, Hemodialysis, Incidence, Reverse epidemiology, Black people

\section{Background}

Cardiovascular disease (CVD) is the leading cause of death in maintenance Hemodialysis (HD), accounting for $40 \%-50 \%$ of all-cause mortality [1]. Stroke is the leading cause of cardiovascular mortality in patients with End Stage Renal Disease (ESRD) [1, 2]. Previous studies from developed countries reported 2- to 10-fold increased risk of stroke in dialysis patients compared to the general population [1]. This propensity for stroke has been attributed to the coexistence of traditional risk

\footnotetext{
* Correspondence: yannicknlandu@yahoo.fr

${ }^{1}$ Nephrology Unit, Department of Internal medicine, Faculty of Medicine, University of Kinshasa Hospital, University of Kinshasa, BP 123 Kinshasa, Democratic Republic of Congo

Full list of author information is available at the end of the article
}

factors, such as hypertension and diabetes mellitus and those specifics to ESRD and dialysis, such as accelerated calcify arteriosclerosis, effects of uremic toxins, dialysis techniques, vascular access, and use of anticoagulants to maintain flow in the extracorporeal circuit $[3,4]$. However, the relative contribution of these factors to the increased cardiovascular risk of dialysis patients remains a matter of debate $[5,6]$. While some studies report a greater contribution of traditional risk factors [3, 5, 7], others underscore a more important role of factors specific to uremic or HD technique $[5,6,8]$ or the conjunction of traditional and dialysis specific risk factors $[5,6,9]$. Hence, it seems rationale for each setting offering maintenance HD to evaluate the burden of stroke and the 
relative contribution of associated risk factors to inform policy makers and care providers.

Democratic Republic of the Congo (DRC), like other sub-Saharan African countries is experiencing an ever growing prevalence of non-communicable disease among which chronic kidney disease and end-stage renal disease [10]. Since 2007, HD centers mainly located in Kinshasa, the DRC capital city are providing maintenance HD. However, the burden of stroke and associated risk factors has not yet been evaluated. Therefore, we designed the present historical cohort study to estimate the incidence rates of stroke in patients undergoing $\mathrm{HD}$ and identify associated risk factors.

\section{Methods}

Patients were recruited from two dialysis centers (Ngaliema Medical Center's Hospital and General Hospital of Kinshasa) between January 1, 2010 and December 31, 2013. All patients aged $\geq 18$ years old who received maintenance HD therapy for more than 3 months were included in the study. The follow-up period for each patient covered from their HD start date until its termination due to death, transplant and shift to peritoneal dialysis, transfer to another center or closing of the study. This is a historical cohort study. Baseline demographic data include age, gender, 24-h urine output, primary cause of ESRD, lifestyle habits (tobacco, alcohol), history of diabetes, hypertension, obesity, Hyperuricemia, hypercholesterolemia or CVD, and biochemical parameters, including hemoglobin, serum albumin, serum calcium, serum phosphorus, total cholesterol, serum Creactive protein (CRP), serum uric acid, serum urea nitrogen, serum creatinine, and dialysis dose as KT/ V. These data were obtained at the start of HD. All parameters were measured in the central laboratory of both hospitals. The study major outcome was stroke, which is defined as a focal neurological deficit of cerebrovascular persisting for $>24$ h diagnosed as an ischemic or hemorrhagic stroke by computed tomography (CT). The diabetes diagnosis at the initiation of dialysis was based on diagnostic criteria from the American Diabetes Association [11]. Hypertension was recorded if the patient was taking any antihypertensive drug or had two separate blood pressure measurements $\geq 140 / 90 \mathrm{mmHg}$. Obesity was defined by a BMI $\geq 30 \mathrm{Kg} / \mathrm{m}^{2}$. Hyperuricemia and hypercholesterolemia was recorded respectively if the patient had an acid uric serum $\geq 7 \mathrm{mg} / \mathrm{dl}$ and a total cholesterol serum $\geq 200 \mathrm{mg} / \mathrm{dl}$. Smoking was defined by a consumption of at least 1 cigarette / day for 5 years or more (active smoking) or withdrawal for less than 5 years (past smoking). Alcoholism was defined by a consumption of at least $20 \mathrm{~g}$ of alcohol / day or $>2$ glasses of beer / day for at least one year. The presence of pre-dialysis comorbid conditions, including history of stroke was determined by a review of medical chart. Cardiovascular Disease (CVD) retrieved from medical chart included left ventricular hypertrophy (LVH), myocardial infarction, atherosclerotic heart disease, cardiomyopathy, cardiac arrhythmia, cardiac arrest, congestive heart failure, cerebrovascular accident, ischemic and peripheral vascular disease. Body mass index (BMI) was calculated as weight $(\mathrm{kg})$ divided by height $(\mathrm{m})$ squared. The pulsed pressure (PP) was calculated as follows: $\mathrm{PP}=$ SBP-DBP. The mean arterial pressure (MAP) as follows: $\mathrm{MAP}=\mathrm{DBP}+(\mathrm{SBP}-\mathrm{DBP}) / 3 . \mathrm{KT} / \mathrm{V}$ was measured by an Online Clearance Monitor (OCM) (Fresenius Healthcare Ltd). The phosphocalcic product was calculated by the product between phosphoremia $(\mathrm{mg} / \mathrm{dl})$ and serum calcium also ( $\mathrm{mg} / \mathrm{dl}$ ).

Summary statistics were presented as percentages for categorical data, mean \pm standard deviation for approximately normally distributed continuous variables, and median for skewed continuous variables. According to stroke events, patients were also divided into two groups: stroke and non-stroke. Characteristic differences between two groups were tested by using the chi-square test for categorical variables, Student's t-test for approximately normally distributed continuous variables, and the non-parametric Mann-Whitney test for skewed continuous variables. The cumulative incidence of stroke was determined using the Kaplan Meier method. Variables found to be significant were used in logistic analysis model using an SAS package. The dependent binary variable in this model was presence (1) or absence (0) of stroke. Statistical significance was defined as $p<0.05$. Statistical analyses were performed using SPSS 17.0 for Windows.

The study protocol was approved by the Clinical Research Ethics Committee of Public Health's School at the University of Kinshasa (Kinshasa, DRC). Since this is a retrospective study, written consent was not required.

\section{Results}

Characteristics of the patients at the start of the study are shown in Table 1. A total of 191 patients were registered (129 males and 62 females, sex ratio 2.1) and the median period of follow up on HD was 5.8 months. The mean age of patients was $52.3 \pm 12.3$ years ranging from 15 to 76 years. It was $54.0 \pm 12.2$ years in male and $48.7 \pm 11.9$ years in female. The mean SBP of patients was $155.7 \pm 27.6 \mathrm{mmHg}$. The main cardiovascular risk factors were anemia (93.7\%), hypertension (85.3\%), LHV (44.1\%) and diabetes mellitus $(41,4 \%)$. The percentage of patients taking aspirin, statin and various types of antihypertensive agents was respectively $28.3,34.2$ and $85.3 \%$. The primary cause of ESRD was Diabetes (64 patients, 33.5\%) followed by Glomerular disease (54 patients, 28.3) and Hypertension (48 patients, 25.1\%). In 122 patients (63.9\%) dialysis was done 2 times per week 
Table 1 Comparative baseline characteristics of patients with and without stroke

\begin{tabular}{|c|c|c|c|}
\hline Variables & $\begin{array}{l}\text { Non Stroke } \\
n=179\end{array}$ & $\begin{array}{l}\text { Stroke } \\
n=12\end{array}$ & $p$ \\
\hline \multicolumn{4}{|l|}{ Demographics } \\
\hline Age $\geq 55$ years, $n(\%)$ & $89(49,7)$ & $6(50,0)$ & 0,985 \\
\hline Male, n(\%) & $117(65,4)$ & $12(100,0)$ & 0,013 \\
\hline Duration of HD (month) & $5,4(3-37)$ & $6,2(3-48)$ & 0,560 \\
\hline Body mass index $\left(\mathrm{Kg} / \mathrm{m}^{2}\right)$ & $24,8 \pm 4,8$ & $22,9 \pm 3,5$ & 0,318 \\
\hline \multicolumn{4}{|l|}{ Etiologies of renal disease } \\
\hline - Diabetic nephropathy & $61(34,1)$ & $3(25.0)$ & 0,519 \\
\hline - Chronic glomerulonephritis & $53(29.6)$ & $1(8.3)$ & 0.113 \\
\hline - Hypertensive & $44(24.6)$ & $4(33.3)$ & 0.499 \\
\hline$\cdot$ HIVAN & $7(3.9)$ & $1(8.3)$ & 0.044 \\
\hline - Polycistic Kidney Disease & $3(1.7)$ & 0 & 0.651 \\
\hline - Obstructive uropathy & $9(5.0)$ & $1(8.3)$ & 0.619 \\
\hline - Chronic pyelonephritis & $2(1.1)$ & $1(8.3)$ & 0.052 \\
\hline \multicolumn{4}{|l|}{ Comorbidities } \\
\hline - Cardiovascular diseases (CVD) & 89 (49.7) & $7(58.3)$ & 0.391 \\
\hline - History of cancer & $10(5.6)$ & $2(16.3)$ & 0.017 \\
\hline$\cdot$ HIV & $9(5.0)$ & $2(16.7)$ & 0.015 \\
\hline - Rhumatismal disease & $17(9.5)$ & $2(16.7)$ & 0.340 \\
\hline \multicolumn{4}{|l|}{ Laboratory variable } \\
\hline Serum creatinine (mg/dl) & $13,9 \pm 8,8$ & $14,3 \pm 5,9$ & 0,901 \\
\hline $\mathrm{GFR}, \mathrm{ml} / \mathrm{min} / 1.73 \mathrm{~m}^{2}$ & $6,06 \pm 3,3$ & $5,17 \pm 2,3$ & 0,363 \\
\hline Serum urea nitrogen (mg/dl) & $219,7 \pm 10,2$ & $269,2 \pm 12,2$ & 0,105 \\
\hline Serum uric acid (mg/dl) & $8,0 \pm 2,9$ & $8,87 \pm 3$ & 0,460 \\
\hline Calcemia (mEq/l) & $4,3 \pm 0,7$ & $4,1 \pm 0,6$ & 0,272 \\
\hline Serum phosphorus (mg/dl) & $5,0 \pm 2,3$ & $4,6 \pm 1,6$ & 0,692 \\
\hline $\operatorname{CaxP}\left(\mathrm{mg}^{2} / \mathrm{dl}^{2}\right)$ & $44,1 \pm 19,4$ & $34,4 \pm 16,8$ & 0,335 \\
\hline Total cholesterol (mg/dl) & $187,8 \pm 63,4$ & $164,2 \pm 56,2$ & 0,551 \\
\hline Serum albumin $(g / l)$ & $37,6 \pm 8,4$ & $37,4 \pm 5,9$ & 0,227 \\
\hline Total protein (g/l) & $65,5 \pm 12,2$ & $68,3 \pm 9,8$ & 0,550 \\
\hline Hemoglobin(g/dl) & $8,1 \pm 1,9$ & $8,5 \pm 2,1$ & 0,519 \\
\hline CRP (mg/l) & $6,3(4,1-12,0)$ & $6,5(5,0-12,0)$ & 0,029 \\
\hline 24 h-proteinuria (g) & $1,7(1,2-2,2)$ & $1,5(1,3-2,3)$ & 0,999 \\
\hline \multicolumn{4}{|l|}{ Risk factors for CVD } \\
\hline Hypertension, n(\%) & $155(86,6)$ & $8(66,7)$ & 0,008 \\
\hline Systolic blood pressure (mm Hg) & $156,9 \pm 27,7$ & $136,8 \pm 19,0$ & 0,014 \\
\hline Diastolic blood pressure(mm Hg) & $86,8 \pm 18,9$ & $79,2 \pm 17,0$ & 0,174 \\
\hline Mean arterial pressure $(\mathrm{mm} \mathrm{Hg})$ & $110,0 \pm 19,8$ & $98,4 \pm 16,3$ & 0,044 \\
\hline Pulse Pressure (mm Hg) & $70,5 \pm 21,2$ & $56,8 \pm 15,1$ & 0,029 \\
\hline Diabetes Melitus, n(\%) & $76(42,5)$ & $3(25,0)$ & 0,189 \\
\hline Obesity, n(\%) & $32(17,9)$ & $4(33,3)$ & 0,169 \\
\hline Prior stroke, n(\%) & $32(17,9)$ & $3(25,0)$ & 0,038 \\
\hline Anemia, n(\%) & $168(93,9)$ & $11(91,7)$ & 0,552 \\
\hline
\end{tabular}

Table 1 Comparative baseline characteristics of patients with and without stroke (Continued)

\begin{tabular}{llll}
\hline LVH, n(\%) & $71(42,8)$ & $7(63,6)$ & 0,015 \\
Hyperuricemia, n(\%) & $32(17,9)$ & $3(25,0)$ & 0,038 \\
Hypercholesterolemia, n(\%) & $44(24,6)$ & $2(16,7)$ & 0,414 \\
Tabac, n(\%) & $15(8,4)$ & $0(0,0)$ & 0,363 \\
Alcohol, n(\%) & $34(19,0)$ & $4(33,3)$ & 0,197 \\
KTN & & & \\
$<1,3$ & $46(43.0)$ & $3(37.5)$ & 0.533 \\
$\geq 1.3$ & $61(57.0)$ & $5(62.5)$ & \\
\hline
\end{tabular}

and in 49 patients $(42.6 \%) \mathrm{KT} / \mathrm{V}$ was inferior to 1.3 . During the four-year study period, we recorded 12 cases (only men) of stroke with intra-cerebral hemorrhage $(\mathrm{ICH})$ and ischemic subtypes in $4(33,3 \%)$ and $8(66,7 \%)$ patients, respectively. The cumulative incidence of stroke increased linearly only in the first year and reaching approximatively $13 \%$ after 4 years. CRP levels were higher in the presence of stroke than in the absence [6.5 $(5.0-12.0)$ vs. 6.3 (4.1-12.0); $p=0.029]$. Compared to patients without stroke, those with stroke had a significantly higher proportion of male subjects (100 vs. $65.4 \%$; $p=0.013$ ), Hyperuricemia ( 25 vs. $17.9 \% ; P=0.038$ ), LVH (63.6 vs. $42.8 \%, p=0.015)$ and prior stroke history (25 vs. $17.9 ; p=0.038$ ); however, they had a significantly lower proportion of Hypertension (66.7\% vs. $86.6 \%$, $p=0.008)$. SBP, PP and MAP were in average lower in the presence of stroke. The results of multiple logistic analyses are shown in Table 2. Absence of Hypertension was identified as a significant predictor of stroke (OR 5.7; 95\%CI 1.52-21.42). The incidence of stroke in the whole group as well as in hypertensive and nonhypertensive patients was $7.4,0.55$ and 2.31 per 1000 patients-months, respectively (Figs. 1 and 2).

\section{Discussion}

The incidence of stroke in this study was 7.4 per 1000 patient-months. This observation corroborates data from the literature reporting a higher incidence of stroke in chronic hemodialysis compared to the general population [3-8]. Indeed, the incidence of stroke observed in this study is higher than that of 1.123 per 1000 patientyears reported in a meta-analysis of some available African studies [12]. This incidence is also higher than that of 0.8 per 1000 patient-years in African hospital settings and those in chronic hemodialysis patients in Europe and Asia who varied between 17 and 74 per 1000 patient-years [3-8]. The propensity of chronic hemodialysis patients to develop more strokes relies mainly upon the acceleration of the dynamic process of atherosclerosis already initiated by uremia and its underlying cause [13]. Indeed, hemodialysis accelerates, 
Table 2 Multiple logistic analysis of predictors of stroke in chronic hemodialysis population

\begin{tabular}{|c|c|c|c|c|c|c|c|c|}
\hline Predictors & $\mathrm{n}$ & person-months & Number of stroke & $\begin{array}{l}\text { Incidence of stroke } \\
\text { per } 100 \text { P-M }\end{array}$ & OR unadjusted (IC95\%) & $p$ & OR adjusted (IC95\%) & $p$ \\
\hline \multicolumn{9}{|c|}{ Hypertension, n(\%) } \\
\hline$-\mathrm{No}$ & 28 & 61 & 4 & 6,56 & $4,39(1,30-14,87)$ & 0,017 & $6,11(1,69-21,99)$ & 0,006 \\
\hline - yes & 163 & 441 & 8 & 1,81 & 1 & & 1 & \\
\hline \multicolumn{9}{|l|}{ LVH } \\
\hline$-\mathrm{No}$ & 99 & 278 & 4 & 1,44 & 1 & & 1 & \\
\hline - Yes & 78 & 187 & 7 & 3,74 & $2,97(0,86-10,24)$ & 0,085 & $3,43(0,96-12,32)$ & 0,058 \\
\hline \multicolumn{9}{|l|}{ Prior Stroke } \\
\hline$-\mathrm{No}$ & 156 & 405 & 9 & 2,22 & 1 & & 1 & \\
\hline - Yes & 35 & 97 & 3 & 3,09 & $1,29(0,35-4,79)$ & 0,707 & $0,81(0,17-3,94)$ & 0,794 \\
\hline
\end{tabular}

through oxidative stress and subsequent inflammation, pre-existing remodeling of all vascular beds especially cerebral vessels $[13,14]$. The particular vulnerability of brain vessels to the deleterious effects of uremia and hemodialysis is related to the structural and functional similarities between renal glomerular microcirculation and cerebral microcirculation $[15,16]$. Indeed, the large extra-cerebral arterial trunks (aorta and carotids) play an important role of shock absorber ("Windkessel effect") to modulate hemodynamic stress from the heart to the brain $[15,16]$. Similar to renal glomerular microcirculation, the peculiar nature of cerebral microcirculation, characterized by high blood flow and low vascular resistance, makes the brain vulnerable to pulsatory hemodynamic stress during each cardiac cycle $[15,16]$. With the acceleration of atherosclerosis through uremia and hemodialysis, the wall of the central elastic arteries (aorta and carotid arteries) loses its elasticity and becomes rigid, resulting in loss of shock function and direct transmission from hemodynamic stress to cerebral microcirculation $[15,16]$. This will result in structural and functional lesions responsible

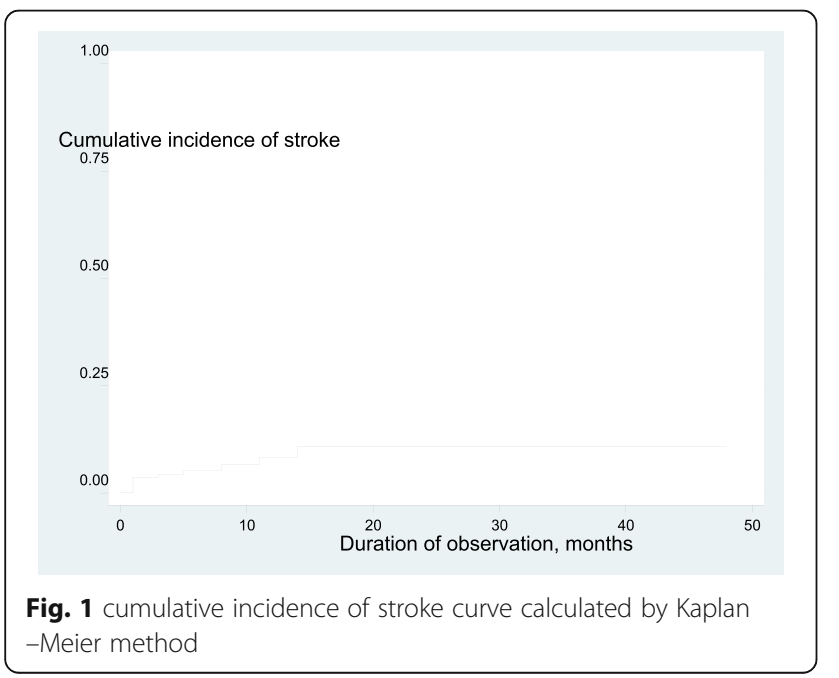

for a wide range of subclinical and clinical conditions including stroke $[15,16]$. Apart from these structural similarities, the high incidence of stroke in chronic hemodialysis patients can also be explained by several other factors including the coexistence of uremic (anemia, hyperphosphorémie, ADMA retention and NO inhibitor) and traditional risk factors as well as the passive selection by hemodialysis of an elderly population with multiple comorbidities $[4,6]$.

In the present study, non-hypertensive patients are at increased risk of stroke suggesting a paradoxical protective effect of elevated blood pressure in chronic hemodialysis. This observation disagrees with the high risk of stroke conferred by elevated blood pressure in the general population. Indeed, INTERSTROKE Study shows that hypertension is the main risk factor for all stroke subtypes, particularly among young Africans who develop stroke while ignoring their hypertensive status [17]. The apparent paradoxical protection afforded by elevated blood pressure against stroke observed in the present study can be partly explained by the phenomenon of reverse epidemiology of

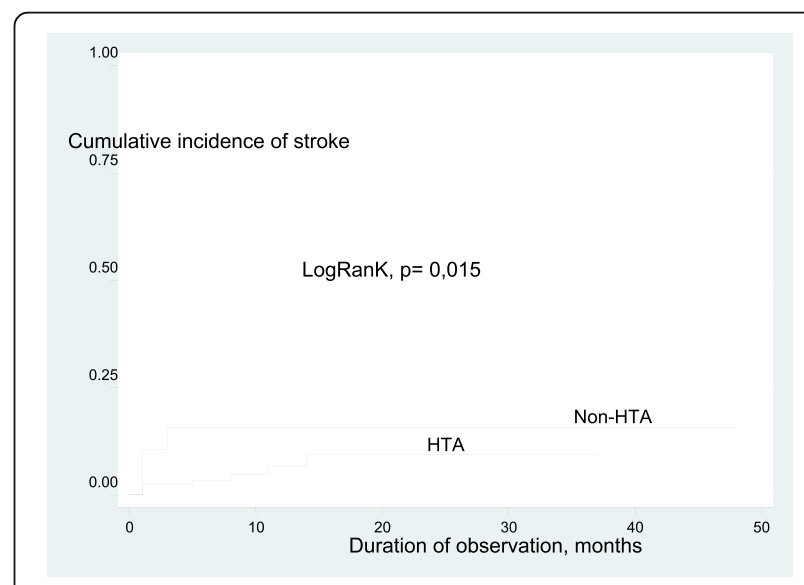

Fig. 2 Cumulative incidence of stroke curve in HTA and non HTA group by Kaplan-Meier method 
traditional risk factors reported both in ESRD and chronic dialysis [18, 19]. This inverse relationship does not necessarily mean that the principles of vascular physiopathology of atherosclerosis are different in patients with ESRD under dialysis; It seems rather to indicate the existence of additional and more powerful risk factors such as inflammation and malnutrition which apparently alter the relationship between traditional risk factors and outcomes in dialysis patients [18, 19]. This phenomenon, not specific to chronic kidney disease and dialysis, is also reported in chronic conditions such as chronic heart failure, cancer and chronic infections like HIV/AIDS $[18,19]$. Another plausible explanation of the apparent paradox can be systolic cardiac dysfunction $[18,19]$. Indeed, hypertension-induced cardiac failure is associated with low blood pressure or hypotension that is both a marker of cardiac disease severity and a powerful predictor of morbidity and mortality [18, 19]. Low blood pressure in uremic patients can also be a reflection of autonomic neuropathy that, in turn, is a marker for more severe uremic complications [18, 19]. Apart from the aforementioned mechanisms underlying reverse epidemiology of hypertension, more recent studies have suggested this phenomenon to be mainly due to inadequacy of peridialytic BP recordings per se to describe the true $\mathrm{BP}$ load, rather than a true U-shaped relationship of BP with cardiovascular morbidity and mortality [20]. Indeed, peridialytic BP recordings are not made for diagnostic reasons but to exploit a major hemodynamic metric like BP in order to assess cardiovascular stability before, during and immediately after the dialysis procedure. Thus, using these readings to diagnose hypertension, assess the success of antihypertensive treatment or examine future cardiovascular risk could be inherently hazardous [20].In contrast to the unclear association of peridialytic BP recordings with allcause and cardiovascular mortality, recent prospective studies have shown that interdialytic BP recorded either at home or by ambulatory BP monitoring (ABPM) associates more closely with mortality and cardiovascular events, as it is documented for the general population [20]. In practice, these data suggest that there is probably no benefit to recommended achieving normal $\mathrm{BP}$ in dialysis. Reverse epidemiology has misleading relevance on dialysis management. The high early mortality universally associated with low baseline BP figures does not contradict the need to achieve normal BP in dialysis patients to reduce longterm cardiovascular mortality. Besides, the eventual noxious/beneficial role of antihypertensive medications in dialysis patients needs to be investigated.

\section{Limitations}

Our study has some limitations that must be acknowledged. First, our results cannot be generalized to all hemodialysis patients since it is not a multicentric study. Second, the study design based on a "post-hoc analysis" of the data for another purpose does not identify the different characteristics (duration, severity ...) of the stroke which was not the main target of the study. Third, the small sample size does not give enough power to statistical tests to identify potential associations between variables of interest.

\section{Conclusion}

Incidence of stroke is higher in Congolese patients receiving hemodialysis with non-hypertensive patients having the highest risk suggesting the reverse epidemiology of elevated blood pressure in chronic hemodialysis.

\section{Abbreviations}

BMI: Body Mass Index; CRP: C Reactive Protein; CT: Computed tomography; CVD: Cardiovascular Disease; DBP: Diastolic Blood Pressure; DRC: Democratic republic of Congo; ESRD: End Stage Renal Disease; HD: Hemodialysis; ICH: Intra-Cerebral hemorrhage; LVH: Left Ventricular hypertrophy; MAP: Mean Arterial Pressure; OCM: Online Clearance Monitor; SAS: Statistical Analysis Software; SBP: Systolic Blood Pressure

\section{Acknowledgements \\ The authors gratefully thank the staff of Ngaliema Medical Center's Hospital and General Hospital of Kinshasa for allowing the conduct of the study.}

\section{Funding}

This work received no financial assistance from any funding agency in the public, commercial, or non-profit sectors.

\section{Availability of data and materials}

All relevant row data can be freely available to any scientists wishing to use them for non-commercial purposes.

\section{Authors' contributions}

YN, FL and J-RM designed the study, acquired, analyzed and interpreted data, drafted and revised the manuscript. FK, YE, ES, VM, JB, JL, M-NW, AL, CZ, $P A, A N, M B$ and $N N$ analyzed, interpreted data and revised the manuscript. All authors read and approved the final manuscript.

\section{Competing interest}

The authors declare that they do not have any financial interest with the information contained in this paper.

\section{Ethics approval and consent to participate}

The study protocol was approved by the Clinical Research Ethics Committee of Public Health's School (Kinshasa, DRC), number ESP/CE/019/2016. This is a retrospective study and written informed consent was not required.

Consent for publication

Not applicable.

\section{Publisher's Note}

Springer Nature remains neutral with regard to jurisdictional claims in published maps and institutional affiliations.

\section{Author details}

${ }^{1}$ Nephrology Unit, Department of Internal medicine, Faculty of Medicine, University of Kinshasa Hospital, University of Kinshasa, BP 123 Kinshasa, Democratic Republic of Congo. ${ }^{2}$ Nephrology Unit, Department of Internal medicine, General Provincial of Kinshasa Hospital, Kinshasa, Democratic Republic of Congo. ${ }^{3}$ Kinshasa School of Public Health, University of Kinshasa Hospital, University of Kinshasa, Kinshasa, Democratic Republic of Congo. ${ }^{4}$ Radiology Unit, Department of Internal medicine, Faculty of Medicine, University of Kinshasa Hospital, University of Kinshasa, Kinshasa, Democratic Republic of Congo. 
Received: 29 April 2017 Accepted: 22 August 2017

\section{Published online: 30 August 2017}

\section{References}

1. Fu J, Huang J, Lei M, Luo Z, Zhong X, Huang Y, et al. Prevalence and Impact on Stroke in Patients Receiving Maintenance Hemodialysis versus Peritoneal Dialysis: A Prospective Observational Study. PLos ONE 10(10):e0140887. doi:10.1371/Journal.pone.0140887.

2. Iseki K, Fukiyama K. Predictors of stroke in patients receiving chronic hemodialysis. Kidney Int. 1996;50:1672-5.

3. Sozio S, Amstrong P, Coresh J, Jaar B, Fink N, Plantinga L, et al. Cerebrovascular disease incidence, characteristics, and outcomes in patients initiating dialysis: the choices for healthy outcomes in caring for ESRD (CHOICE)study. AJKD. 2009;54:468-77.

4. Power A. Stroke in dialysis and chronic kidney. Blood Purif. 2013;36:179-83.

5. Herrington W, Haynes R, Staplin N, Emberson J, Baigent C, Landray M. Evidence for the prevention and treatment of stroke in dialysis patients. Semin Dial. 2015;28:35-47.

6. Saeed F, Kousar N, Qureshi K, Laurence NT. A review of risk factors for stroke in patients with chronic kidney disease. Journal of Vascular and Interventional Neurology. 2009;2(1):126-31.

7. Belmar L, Angel LM, Laura B, Celestino P, Elena M, Maria $K$, et al. Strokes in patients on Haemodialysis: incidence. Onset Time and Associated Factors Nefrologia. 2014;34(3):347-52.

8. Murray MA, Seliger S, Lakshminarayan K, Herzog CA, Solid CA. Incidence of Stroke before and after Dialysis Initiation in Older Patient. J Am Soc Nephrol 24:1166-1173.

9. Hill RN, Futoba ST, Oke JL, Hirst JA, O'Callaghan CA, Lasserson DS, et al. Global Prevalence of Chronic Kidney Disease - a Systematic Review and Meta-analysis. PLos ONE 11(7): 0158765.doi:10:1371/journal.pone.0158765.

10. Sumaili EK, Krzesinski JM, Zinga CV, Cohen EP, Delanaye P, Munyanga SM, et al. Prevalence of chronic kidney disease in Kinshasa. Results of pilot study from the Democratic Republic of the Congo. Nephrol dial. Transplantation. 2009;24(1):117-22.

11. American Diabetes Association. Position statement, diagnosis and classification of diabetes mellitus. Diabetes Cre. 2008;31(Suppl 1):S55-60.

12. Davies Adeloye. An Estimate of the incidence and Prevalence of Stroke in Africa: A Systematic Review and Meta-analysis. PLos ONE 9(6): e100724. doi: 10.1371/journal.pone.0100724.

13. Walter R, Mischak H, Haller H. Haemodialysis, atherosclerosis and inflammation-identifying molecular mechanisms of chronic vascular disease in ESRD patients. Nephrol Dial Transplant. 2002;17(3):24-9.

14. Bevc S, Hojs R, Ekart R, Hojs-Fabjan. Atherosclerosis in Heamodialysis patients: traditional and non traditional risks factors. Acta Dermatoven APA 2006;15:151-157.

15. Toyoda K. Cerebral small vessel disease and chronic kidney disease. Journal of Stroke. 2015;17(1):31-7.

16. Shoji T, Emoto M, Tabata T, Kimoto E, Shinohara K, Maekawa K, et al. Advanced atherosclerosis in Predialysis patients with chronic renal failure. Kidney Int. 2002:61:2187-92.

17. O'Donnell MJ, Xavier D, Liu L, Zharg H, Chin SL, Rao-Melacini P, et al. Risk factors for ischemic and intracerebral Haemorragic stroke in 22 countries (the INTERSTROKE study): a case control study. Lancet. 2010;376:112-23.

18. Kalantar-Zadeh K, Block G, Humphreys MH, Kopple JD. Reverse epidemiology of cardiovascular risk factors in maintenance dialysis patients. Kidney Int. 2003;63:793-808.

19. Kopple JD. The phenomenon of altered risk factor patterns or reverse epidemiology in persons with advanced chronic kidney failure. Clin Nutr. 2005:81:1257-66

20. Sarafidis PA, Persu A, Agarwal R, Burnier M, de Leeuw P, Ferro CJ, et al. Hypertension in dialysis: a consensus document by European renal and cardiovascular medicine (EURECA) working Group of the European Renal Association-European Dialysis and Transplantation Association (ERA-EDTA) and the hypertension and the kidney working Group of the European Society of hypertension (ESC). Nephrol Dial Transplant. 2017;32:620-40.

\section{Submit your next manuscript to BioMed Central and we will help you at every step:}

- We accept pre-submission inquiries

- Our selector tool helps you to find the most relevant journal

- We provide round the clock customer support

- Convenient online submission

- Thorough peer review

- Inclusion in PubMed and all major indexing services

- Maximum visibility for your research

Submit your manuscript at www.biomedcentral.com/submit 